\title{
PERFIL E INVENTARIO DE PERSONALIDAD DE ESTUDIANTES DE ENFERMERÍA Y SU DESEMPEÑO ACADÉMICO, 2011
}

\author{
Luis Vólmar Quintero MD*, Carlos Alberto Castro MD**
}

\section{Resumen}

Introducción: para ingresar a la educación superior se utilizan criterios de selección cada vez más exigentes con el ánimo de garantizar la excelencia. Las instituciones buscan un perfil determinado para cada programa académico empleando diversos tipos de pruebas psicotécnicas. Objetivo: determinar si la prueba del perfil e inventario de la personalidad de Gordon (P-IPG) es un predictor del desempeño académico en estudiantes de enfermería. Materiales y métodos: estudio de cohorte histórica, la variable dependiente fue el promedio académico de inicio y mitad de la carrera, las variables independientes fueron los resultados de la prueba P-IPG y las características sociodemográficas. Los datos se analizaron en Stata 10. La correlación se calculó con el coeficiente de correlación de Pearson para ajustar un modelo de regresión lineal múltiple. Resultados: se analizaron 202 estudiantes de enfermería, 26\% (51) de colegios de fuera de Bogotá, 49,2\% (99) se graduaron en colegios privados y el resto públicos, habilitaron $80,8 \%$ (84) y $42 \%$ (85) repitieron al menos una materia. Se calculó la correlación entre el promedio inicial y la responsabilidad $(\rho=0,17)$, originalidad $(\rho=0,16)$ y el vigor $(\rho=0,16)$. Se ajustó un modelo de regresión múltiple para los promedios (inicial y mitad de la carrera), con un $\mathbf{R}^{2}=0,10$. Conclusión: hay correlación leve entre los resultados de la prueba de personalidad P-IPG de los estudiantes con el desempeño académico al inicio y mitad de la carrera. Es deseable establecer un perfil que se pueda relacionar con un mejor desempeño académico y profesional.

Palabras clave: P-IPG, rendimiento académico, estudiantes, salud.

Abreviaturas: P-IPG, prueba del perfil e inventario de personalidad de Gordon.

\section{PERSONAL PROFILE INVENTORY OF A GROUP OF NURSING STUDENTS AND ITS CORRELATION WITH ACADEMIC PERFORMANCE, 2011}

\section{Abstract}

Introduction: eligibility criteria for admission to undergraduate education are increasingly demanding in order to ensure excellence. Institutions look for a certain profile of candidates for a particular academic program using various types of psycho-technical tests. Objective: to determine if Gordon Personal Profile-Inventory (GPP-I) test is a predictor of academic performance in nursing students. Materials and Methods: historic cohort study in

Fecha recibido: febrero 5 de 2013 - Fecha aceptado: mayo 7 de 2013

* Médico Psiquiatra, MSc Bioética. Director Oficina de Orientación Universitaria, Profesor Asociado Fundación Universitaria de Ciencias de la Salud, Bogotá DC, Colombia.
** Coordinador de Investigaciones para pregrado y Semilleros de Investigación, Instructor Asociado Fundación Universitaria de Ciencias de la Salud. Bogotá DC, Colombia. 
which the dependent variable was the initial and mid-career grade averages and the independent variables were the GPP-I test results and the social and demographic features. Stata 10 was used for data analysis. Pearson correlation coefficient was used to estimate the correlation to adjust a multiple linear regression model. Results: 202 nursing students were analyzed, $26 \%$ (51) of schools outside Bogotá, $49.2 \%$ (99) graduated from private schools and the rest from public schools, $80.8 \%(84)$ took make-over tests and $42 \%(85)$ repeated at least one course. The correlation between the initial average and dependability $(\rho=0.17)$, innovation $(\rho=0.16)$ and vigor $(\rho=0.16)$ was estimated. A multiple regression model for initial and mid-career grade averages was adjusted, obtaining an $\mathbf{R}^{2}=\mathbf{0 . 1 0}$. Conclusion: a mild correlation between the GPP-I test results and initial and mid-career academic performance of students is evidenced. It would be desirable to establish a profile which can be related to better academic and professional performance.

Key words: P-IPG, academic performance, students, health

\section{Introducción}

En Colombia existen 282 instituciones de educación superior con un incremento anual de cinco nuevas ante el Ministerio Educación Nacional. ${ }^{1}$ Como ha ocurrido en otras naciones, este auge determina la necesidad de criterios de selección cada vez más exigentes con el ánimo de garantizar que sus estudiantes no solo sean superiores en la academia, sino que tengan mejores habilidades y destrezas personales.

Es común que las instituciones busquen un perfil determinado para cada programa académico, el cual se define por criterios tales como los resultados del examen de admisión, la prueba de estado o las calificaciones en los últimos años en la educación media; de igual manera, en algunas se utilizan entrevistas que apoyadas por pruebas psicotécnicas, buscan encontrar los estudiantes que llenen el perfil deseado.

Existen diversos tipos de pruebas psicotécnicas que miden en su gran mayoría habilidades y destrezas, como por ejemplo la capacidad de cálculo, comprensión lectora, razonamiento abstracto y el coeficiente intelectual entre otras. En la actualidad se cree que el rendimiento escolar es una característica multidimensional ${ }^{2}$ que depende de factores no solo de tipo académico sino de los rasgos de personalidad, sobre la base de que estos son bastante estables en el transcurso de la vida de los individuos y pueden predecir sus tendencias comportamentales. Existe un consenso entre autores con respecto a la existencia de cinco factores en la estructura de la personalidad que sirven en los diferentes procesos de evaluación académica o desempeño laboral ${ }^{3}$, estos son: tendencia a estar solo o con otros, amabilidad, responsabilidad, adaptabilidad emocional y apertura a nuevas experiencias. Una de esas pruebas es el denominado perfil e inventario de Gordon (P-IPG), muy utilizado en diversas instituciones de educación superior. Constituye una herramienta para hacer un seguimiento a los estudiantes de mayor riesgo. Se entiende como desempeño académico el resultado medido por una escala numérica, obtenido de una serie de factores que actúan en y desde la persona que aprende una disciplina en particular. Es el resultado del conocimiento suscitado por la actividad educativa del profesor y producido en el alumno; se expresa con una calificación cuantitativa y cualitativa que cuando son consistentes y válidas serán el reflejo del aprendizaje y del logro de determinados objetivos. ${ }^{4}$ Por lo anterior, el presente estudio tiene como objeto determinar la correlación entre la prueba (P-IPG) y el desempeño académico de estudiantes de enfermería de una institución educativa de ciencias de la salud.

\section{Materiales y métodos}

Se realizó un estudio de cohorte histórica; la población a estudio fueron estudiantes que pertenecieran a la facultad de enfermería de una institución de educación superior de Bogotá DC, que hubiesen presentado la prueba psicotécnica P-IPG al inicio de la carrera, admitidos para cursar primer semestre y registrados en el sistema de la universidad (Academusoft), por esta razón se tomaron los semestres cuarto, quinto, sexto, séptimo y octavo. Se excluyeron los que no presentaran 
registro completo de datos. La variable dependiente se definió como el promedio de notas al final del primer semestre y de la mitad de la carrera. Las variables independientes fueron los resultados de la prueba P-IPG (dominio) y las características sociodemográficas. Se realizó una prueba piloto para identificar problemas en la recolección y determinar el rendimiento de los instrumentos. La recolección de los datos se hizo a partir de las hojas de vida de los estudiantes y los registros de notas reportados por el programa Academusoft, datos que después se consignaron en un instrumento estructurado por los autores. Después se digitaron en una base de datos Excel 2010 la cual fue corroborada con la verificación del $10 \%$ de los registros seleccionados en forma aleatoria.

Instrumentos: la prueba de P-IPG es una escala psicotécnica que mide cinco perfiles y cuatro inventarios de personalidad, los cuales se definen a continuación. Ascendencia: caracteriza a individuos verbalmente dominantes, que adoptan un papel activo dentro del grupo. Responsabilidad: capaces de preservar el trabajo que se les asigna. Estabilidad emocional: por lo regular sujetos estables y bastante libres de preocupaciones. Sociabilidad: les gusta estar y trabajar con otras personas. Autoestima: describe características deseables e indeseables del sujeto (pensamientos buenos de sí mismos). Cautela: demuestran cuidado antes de tomar decisiones o actuar. Originalidad: les gusta trabajar en problemas difíciles y poseen curiosidad intelectual. Relaciones personales: demuestran confianza y fe en los demás. Vigor: son enérgicos, les gusta el trabajo con alto ritmo. ${ }^{5}$

Esta escala fue validada en español (México) y se utiliza en la selección y admisión de estudiantes en instituciones de educación superior. Para la variable ocupación se tuvo en cuenta la clasificación sugerida por la Organización de Estados Iberoamericanos (OEI) acogida por el Servicio Nacional de Aprendizaje (SENA). ${ }^{6}$

El estudio fue aprobado por el comité de ética de investigación con seres humanos de la Fundación Universitaria de Ciencias de la Salud. Los resultados del ICFES-Saber PRO fueron categorizados de acuerdo con la clasificación referida por esta misma institución oficial (bajo, menor o igual a 30, medio, 31 a 70 y alto, mayor de 71). Para la categorización de los resultados por rasgo en la P-IPG se definió: muy bajo $=0-20$, bajo $=21-40$, medio $=41-60$, alto $=61-80 \mathrm{y}$ muy alto $=81-100$.

Análisis estadístico: se realizó en el programa Stata 10. Se utilizaron medidas de tendencia central y de dispersión para las variables cuantitativas y para las cualitativas se presentaron frecuencias absolutas y relativas. Se calculó el coeficiente de correlación de Pearson y se ajustó un modelo de regresión lineal múltiple de acuerdo con las variables definidas con antelación.

\section{Resultados}

Se analizaron 202 estudiantes de enfermería que iniciaron estudios en el primer período del 2009, a los cuales se les hizo seguimiento histórico de su actividad académica cumpliendo los criterios de admisión. Las características generales de la población fueron: 8,5\% (17) hombres, $26 \%$ (51) provienen de colegios de fuera de Bogotá, 49,2\% (99) se graduaron en colegios privados y $50,7 \%$ (102) de los públicos, entre los que se incluyeron distritales, nacionales y departamentales. Los resultados del promedio académico del primer semestre se reportan en la Tabla 1.

Tabla I.Características generales y académicas de la población

\begin{tabular}{|l|c|}
\hline Sexo - femenino $n(\%)$ & $185(9 I .5)$ \\
\hline Edad (años) - prom (DE) & $18.2(3)$ \\
\hline ICFES/Saber PRO - prom (DE) & $48,5(6,2)$ \\
\hline Notas de la carrera - prom (DE) \\
\hline inicio (Ier. semestre) & $3,5(0,2)$ \\
\hline mitad (4o. semestre) & $3,6(0,2)$ \\
\hline Habilitación de materias - $n(\%)$ & $104(5 \mathrm{I}, 4)$ \\
\hline Repetición de materia $-n(\%)$ & $85(42)$ \\
\hline Repetición de semestre $-n(\%)$ & $33(16,6)$ \\
\hline
\end{tabular}


En la Tabla 2 se observan las ocupaciones de los padres y madres de los estudiantes teniendo en cuenta la clasificación de la Organización de Estados Iberoamericanos OIE (avalada y aceptada por el SENA), que dentro de sus opciones está las "no clasificables", la cual para padres, 28 correspondían a independiente y 13 a jubilado. De las 80 madres en esta misma clasificación, 57 correspondían a ocupación hogar/ama de casa y 18 laboraban como independientes.

De 104 estudiantes que habilitaron, 80,8\% (84) fue inglés (nivel I, II y/o III), 11,5\% (12) investigación (básica, media y/o cualitativa), 11,5\% (12) expresión oral, 9,6\% (10) legislación en salud y 7,7\% (8) farmacología. 29,8\% (31) habilitaron dos materias, $8,6 \%$ (9) tres y solo una estudiante habilitó cuatro materias. 17,3\% (18) lo hicieron en segundo semestre y $23 \%$ (24) en tercero y cuarto, $42 \%$ (85) repitieron al menos una materia, de los cuales el 36,4\% (31) repitió dos y 10,5\% (9) tres simultáneas. De 33 estudiantes que repitieron semestre 54\% (18) el primero, $27 \%$ (9) segundo, 6\% (2) tercero, $48 \%$ (16) cuarto, 3\% (1) quinto y sexto; es importante resaltar que de estos mismos estudiantes $85 \%$ (28) repitieron un semestre, $24 \%$ (8) dos y $3 \%$ (1) tres semestres.
Con respecto a los resultados de la P-IPG, se observaron altos puntajes en los dominios de cautela, vigor y ascendencia (Tabla 3). Se calculó la correlación entre los promedios (inicial y mitad de carrera) y los diferentes dominios reportados por la prueba P-IPG usando el coeficiente de Pearson, encontrando solo correlaciones leves entre el promedio inicial y la responsabilidad ( $\rho=0,17)$, originalidad $(\rho=0,16)$ y vigor $(\rho=0,16)$; de igual manera se calculó la correlación entre el promedio a mitad de la carrera y los dominios referidos en la prueba psicotécnica, encontrando correlaciones con responsabilidad $(\rho=0.21)$ y vigor $(\rho=0.19)$.

Después se ajustó un modelo de regresión múltiple para los promedios (inicial y mitad de carrera) teniendo en cuenta el sitio del colegio donde se graduó el estudiante, tipo de bachillerato, resultados de las pruebas de estado (ICFES-Saber-PRO), y la ocupación de los padres y las madres, los cuales no mostraron modificaciones en el modelo propuesto reportando bajas correlaciones entre los dominios y los promedios. En la Tabla 4 se muestran los posibles dominios que explican el promedio de mitad de la carrera, sin embargo el $\mathrm{R}^{2}$ fue igual a 0,10 mostrando poca explicación de los datos por el modelo.

\begin{tabular}{|l|c|c|}
\hline \multicolumn{3}{|c|}{ Tabla 2. Ocupación de padres y madres } \\
\hline Ocupaciones - Actividades laborales* & Padres & Madres \\
\hline Finanzas y administración & $\mathrm{n}(\%)$ & $\mathrm{n}(\%)$ \\
\hline Ciencias naturales, aplicadas y relacionadas & $14(6,9)$ & 2 I $(10,4)$ \\
\hline Salud & $19(9,4)$ & $2(0,9)$ \\
\hline Ciencias sociales, educación, administración pública y religión & $3(1,4)$ & $22(10,8)$ \\
\hline Arte, cultura, recreación y deporte & $9(4,4)$ & $13(6,4)$ \\
\hline Ventas y servicios & $2(0,9)$ & $2(0,9)$ \\
\hline Explotación primaria y extractiva & $36(17,8)$ & $32(I 5,8)$ \\
\hline Oficios, operadores de equipo y transporte & $4(1,9)$ & $3(1,4)$ \\
\hline Procesamiento, fabricación y ensamble de bienes & $45(22,2)$ & $1 \mathrm{I}(5,4)$ \\
\hline No clasificables & $1(0,5)$ & $\mathrm{I}(0,5)$ \\
\hline
\end{tabular}

* Clasificación de la Organización de Estados Iberoamericanos OIE (avalada y aceptada por el SENA). 


\begin{tabular}{|c|c|c|c|c|c|}
\hline \multicolumn{6}{|c|}{ Tabla 3. Resultados la prueba P-IPG } \\
\hline \multirow{2}{*}{ Dominio } & \multicolumn{5}{|c|}{ Categorización } \\
\hline & Muy alto n (\%) & Alto n (\%) & Medio n (\%) & Bajo n (\%) & Muy bajo n (\%) \\
\hline Responsabilidad & $135(7 \mid)$ & $36(18,9)$ & $14(7,3)$ & $5(2,6)$ & $0(0)$ \\
\hline Estabilidad emocional & $61(32, I)$ & $71(37,3)$ & $30(15,7)$ & $17(8,9)$ & II $(5,7)$ \\
\hline Sociabilidad & $6(3,1)$ & $40(2 I)$ & $83(43,6)$ & $51(26,8)$ & $10(5,2)$ \\
\hline Cautela & $77(40,5)$ & $63(33,1)$ & $32(16,8)$ & $15(7,8)$ & $3(1,5)$ \\
\hline Originalidad & $51(26,8)$ & $78(4 I)$ & $36(18,9)$ & $24(12,6)$ & $\mathrm{I}(0,5)$ \\
\hline Relaciones personales & $47(24,7)$ & $65(34,2)$ & $53(27,8)$ & $18(9,4)$ & $7(3,6)$ \\
\hline Vigor & $114(60)$ & $54(28,4)$ & $17(8,9)$ & $2(I)$ & $3(1,5)$ \\
\hline Ascendencia & $88(46,3)$ & $52(27,3)$ & $38(20)$ & $10(5,2)$ & $2(1)$ \\
\hline
\end{tabular}

La categorización que se muestra en la tabla fue realizada por los autores de la prueba P-IPG.

\begin{tabular}{|l|c|c|c|c|}
\hline \multicolumn{4}{|c|}{ Tabla 4. Resultados - regresión lineal múltiple } \\
\hline \multicolumn{1}{|c|}{ Dominio } & Coeficiente & Error estándar & T & 3,29 \\
\hline Responsabilidad & 0,0041 & 0,001 & 2,001 \\
\hline Vigor & 0,0022 & 0,001 & $-2,77$ & 0,038 \\
\hline Estabilidad emocional & $-0,0027$ & 0,000 & 30,3 & 0,006 \\
\hline Constante & 3,3603 & 0,11 & 0,000 \\
\hline
\end{tabular}

Variable dependiente: promedio de mitad de carrera $\left(\mathrm{R}^{2}=0,10\right)$.

\section{Discusión}

El ingreso a la universidad para los jóvenes recién graduados del colegio trae cambios abruptos por la metodología de estudio, de trabajo y el ritmo de vida. ${ }^{8} \mathrm{La}$ forma en que percibimos y procesamos la información y la dificultad con que experimentamos la adquisición de nuevo conocimiento, están influenciadas por la personalidad y el estilo de aprendizaje. ${ }^{9}$ Por esta razón, los procesos de selección de los candidatos a la educación superior se basan en los perfiles para cada carrera, que González lo resume en lo que él llama "elección vocacional" la cual hace parte de la autoasignación que hace cada aspirante en la escogencia de su proyecto de vida, relacionada con las habilidades mentales y su estructura de inteligencia. ${ }^{10}$
El proceso de formación de los estudiantes se fundamenta en el rendimiento académico, el cual responde según Jara a la capacidad de respuesta que tiene un individuo a los estímulos, dada por objetivos y propósitos educativos que son evaluados para determinar el aprendizaje que se obtuvo. ${ }^{8}$ Cuando hablamos de un buen rendimiento, muchas instituciones de educación superior (IES) toman las notas (numéricas) con puntos de corte que varían de acuerdo con el establecimiento educativo. A partir de estos criterios se han determinado algunos factores que pueden influir en el desempeño académico durante la carrera como los antecedentes académicos del colegio y los factores socioeconómicos (ingreso económico, entorno social, composición familiar). 
Britan en un estudio descriptivo analizó 66 estudiantes de ciencias de la salud, que clasificó por medio de una prueba psicotécnica (MBT) al ingreso de la carrera, la cual evaluaba las preferencias psicológicas de los sujetos según Carl Jung en asimiladores (44\%) y convergentes (35\%). Estas categorías mostraban la capacidad de un estudiante para reflexionar información y luego aplicarla reconociendo patrones que estaban en relación directa con un buen desempeño académico. También encontró que estas características se asociaban más con las mujeres que con los hombres. ${ }^{9}$ Jara en su estudio de 44 sujetos, también de ciencias de la salud, identificó una relación directamente proporcional con el rendimiento académico a partir de factores tales como el género, el rendimiento escolar previo y los factores económicos familiares, los cuales varían de acuerdo con las capacidades propias de autoaprendizaje, grado de motivación, ausentismo, repetición de materias, semestres y deserción. . $^{811,12}$

En nuestro estudio no se encontraron relaciones estadísticas significativas entre los antecedentes del lugar de origen del estudiante, el tipo de colegio del cual se habían graduado, la repetición/perdida y la habilitación de materias frente a los dominios de la prueba ni con los promedios académicos.

Con respecto a los resultados de P-IPG, no se evidenció una alta correlación con los resultados académicos, aunque de manera leve si se observa al momento del inicio de la carrera con los dominios de la prueba denominados responsabilidad, entendida esta como capacidad de perseverar en el trabajo asignado y confiabilidad; además la originalidad se relacionó en forma leve, la cual se define como el gusto por trabajar en problemas difíciles y por último el vigor que es el gusto por el trabajo con alto ritmo. A la mitad de la carrera persiste la correlación con responsabilidad y vigor. Es importante mencionar que estas características se incluyen dentro de los perfiles que algunos autores han referido como relevantes para un profesional en enfermería.
Es interesante además resaltar que la ocupación de los padres en su gran mayoría se encuentra en ventas y servicios, y en actividades tales como operadores de equipos y transporte, mientras que las de la madre se evidencian otras ocupaciones como finanzas, administración y salud. Sin embargo, para el sistema de clasificación no se tienen en cuenta las amas de casa ni los jubilados y/o pensionados que representan el $23 \%$ y $39,6 \%$.

Es importante señalar que las actividades de los padres y su nivel académico están relacionadas con el rendimiento de los estudiantes y la posibilidad de continuar con estudios de postgrados. ${ }^{13}$ Con respecto a la repetición y habilitación de materias, es relevante mencionar que la materia con la que más se presentaron dificultades académicas fue inglés que podría evidenciar el bajo nivel colegial con que los estudiantes se enfrentan a una IES.

\section{Conclusiones}

Existe una correlación leve entre los resultados de la prueba de personalidad P-IPG de los estudiantes de enfermería con el desempeño académico a mitad de la carrera, sin embargo, se observa una leve correlación (baja explicación de la variabilidad entre la prueba con respecto al desempeño académico) con los dominios de responsabilidad, originalidad y vigor que son también características deseables, entre otras, para personas que se dedican a actividades relacionadas con la salud. Se considera que a pesar de los resultados obtenidos, sigue siendo anhelado, de acuerdo con la literatura,${ }^{2}$ establecer un perfil del estudiante con parámetros claros que puedan predecir un desempeño académico superior y por ende con un mejor profesional. Se hace recomendable ampliar este estudio en otras facultades para que sirva como elemento comparativo. 


\section{Referencias}

1. Colombia. Ministerio de Educación Nacional. Deserción por cohorte según Nivel de formación. Sistema para la Prevención de la Deserción de la Educación Superior. Bogotá: El Ministerio; 2011.

2. Rodríguez Pichardo CM, Ávila Ortega A, González M, Heredia Escorza Y. Perfil psicosocial y uso de las tecnologías de la información y la comunicación de alumnos con promedios académicos altos y minimos de la modalidad educativa presencial en un contexto mexicano. REDIE. 2008;10(2):1-19.

3. Puentes A, Pulido N. Personalidad y ausentismo en trabajadores de servicios generales de una universidad pública. Hacia la Promocion de la Salud. 2009;15(1):29-40

4. Castro A. Estilos de personalidad, afrontamiento e inteligencia como predictores de las trayectorias acad,micas de cadetes en una institución militar. Anu. Psicol. 2005; 36(2):197-210.

5. Gordon LV. Pérfil - Inventario de la Personalidad. $2^{\mathrm{a}}$ ed. México : Manual moderno; 1994.

6. OEI. Clasificación nacional de ocupaciones C.N.O: una herramienta para gestión de empleo y formación [Monografía en Internet]. Bogotá: SENA; 1996 [citado 19 Jun 2013]. Disponible en: http://www.oei.es/oeivirt/fp/cuad2a03.htm.
7. ICFES. Clafisiciación de los resultados SaberPro. Bogotá: ICFES; 2011.

8. Jara D. VH, Gordillo G., Guerra G., León I., Arroyo C., Figueroa M. Factores influyentes en el rendimiento académico de estudiantes del primer año de medicina. An Fac med. 2008;69(3):193-7.

9. Britan M, Lafuente M, Zuñiga D, Viviani P, Beltrán MC. ¿Influyen las carac terísticas psicológicas y los estilos de aprendizaje en el rendimiento académico de los estudiantes de medicina? Un estudio retrospectivo. Rev Méd Chile. 2004;132(9):1127-36.

10. Gonzales G, Castro Solano A, Gonzalez F. Perfiles aptitudinales, estilos de pensamiento y rendimiento académico. Anu. Investig. 2008;15:33-41.

11. Frischenschlager O, Haidinger G, Mitterauer L. Factors associated with academic success at Vienna Medical School: Prospective survey. Croat Med J. 2005;46(1):58-65.

12. Mills C, Heyworth J, Rosenwax L, Carr S, Rosenberg M. Factors associated with the academic success of first year Health Science students. Adv Health Sci Educ Theory Pract. Adv Health Sci Educ Theory Pract. 2009 May;14(2):205-17

13. Corengia A, Mesurado B, Redelico F. Las aptitudes educacionales y su relación con el rendimiento académico y la deserción: un estudio exploratorio. Rev. Int. Estud. Polit. Educ. 2006(5):75-83. 